Juan Pedro Alonso ${ }^{1}$

\title{
Cuidados paliativos: entre la humanización y la medicalización del final de la vida
}

\author{
Palliative care: \\ between humanization and medicalization at the end of life
}

\footnotetext{
${ }^{1}$ Instituto de

Investigaciones Gino Germani, Facultad de

Ciencias Sociales, Universidad de Buenos Aires. Pte. J.E. Uriburu 950, $6^{\circ}$. C1114AAD Buenos Aires Argentina. juan_alonso79@hotmail.com
}

\begin{abstract}
This paper analyzes the palliative care of terminal patients, examining the tensions between the humanization of care and the progressive medicalization at the end-of-life situation. The research upon which the article is based adopts a qualitative methodological approach derived from interviews with professionals and patients and ethnographic observations in a palliative care unit in the City of Buenos Aires, Argentina. The article describes the configuration of personalized and comprehensive health care based on the core values of more humanized endof-life care promoted by palliative care supporters. Similarly, the paper analyzes how these practices are assisted by progress in the medicalization process in which the dimension of care is considered less an unprofessional area of medical practice than an area of care in which specific technical skills and know-how are employed. The articles explores how instead of being divergent, the logic of care and medicalization work in a complementary fashion.
\end{abstract}

Key words Palliative care, End of life, Care, Medicalization
Resumen El artículo analiza la atención de pacientes terminales en servicios de cuidados paliativos, explorando las tensiones entre la humanización del cuidado y el avance de la medicalización en el final de la vida. La investigación en que se apoya el artículo sigue una estrategia metodológica cualitativa, basada en entrevistas con profesionales y pacientes y en observaciones etnográficas en un servicio de cuidados paliativos de la Ciudad de Buenos Aires, Argentina. El artículo describe la configuración de un cuidado personalizado e integral, base de la propuesta de la disciplina de brindar una atención más "humana" en el final de la vida. Asimismo, se analiza la forma en que éstas prácticas están mediadas por el avance de procesos de medicalización, en las que el cuidado es considerado menos un aspecto "no profesional" de la práctica médica que un área de atención sobre la que se afirman habilidades y saberes técnicos específicos. El artículo evidencia cómo las lógicas del cuidado y de la medicalización, más que lógicas opuestas, funcionan de forma complementaria.

Palabras clave Cuidados paliativos, Fin de vida, Cuidado, Medicalización 


\section{Introducción}

Producto de cambios culturales, demográficos y epidemiológicos, y de la profesionalización y los avances en el campo médico, durante el siglo XX se consolidó en las sociedades occidentales la tendencia a que la muerte y el final de la vida ocurran bajo la esfera de la medicina ${ }^{1-4}$. A partir de una serie de innovaciones técnicas (como la ventilación pulmonar, la resucitación cardiopulmonar y la nutrición artificial, entre otras) se inaugura a partir de las décadas del ' 50 y el ' 60 en los EE.UU la terapia intensiva, modalidad asistencial que modificó de manera significativa la gestión médica de estos procesos, posibilitando mantener órganos vitales por medios tecnológicos y la prolongación artificial de la vida ${ }^{5-8}$.

En la segunda mitad del siglo XX comenzaron a manifestarse voces críticas de la medicalización de la muerte y el morir y las consecuencias sociales de estos procesos ${ }^{2,3,9-13}$. Asimismo, comienzan a desarrollarse movimientos e iniciativas (tanto dentro como fuera del campo de la medicina) que promueven formas "más humanas" de abordar el final de la vida. En este sentido, la emergencia y desarrollo del movimiento de los hospices y los cuidados paliativos ${ }^{14}$, los movimientos a favor de la eutanasia y del derecho a morir con dignidad ${ }^{15,16}$, el desarrollo de la bioética y el mayor énfasis en el respecto de la autonomía de los pacientes ${ }^{17-19}$ contribuyeron a cuestionar la forma de gestionar la muerte en contextos médicos.

En este artículo se analiza la tensión entre las iniciativas de "humanización" de los cuidados y los procesos de medicalización en el final de la vida, tomando como punto de partida una de estas iniciativas: los cuidados paliativos, disciplina médica especializada en la atención de pacientes terminales. Herederos del movimiento de los hospices, los cuidados paliativos se presentan como una crítica a la forma tecnificada e impersonal de gestionar la muerte en los ámbitos médicos, a partir de un abordaje interdisciplinario que propone una atención integral, basada en el control de síntomas físicos, el apoyo psicosocial y espiritual ${ }^{14,20}$.

Puntualmente, el artículo aborda la tensión entre "humanización" y medicalización del final de la vida tomando como eje de análisis las nociones de cuidado (personalizado, integral) propuestas por esta disciplina. Para sus promotores y algunos autores, esta disciplina supone una respuesta al sufrimiento que apela al acompañamiento y a la singularidad del paciente, comprometido con un uso responsable de la tecnología $a^{21,22}$. Desde posturas críticas, sin embargo, se ha señalado el avance de la medicalización y el poder de la medicina en el periodo final de la vida que ha supuesto la emergencia y desarrollo de una especialidad médica sobre el proceso de morir. Entre las críticas, se ha señalado la creciente medicalización de los cuidados paliativos, operada con la profesionalización de la disciplina ${ }^{23-25}$. Estos autores señalan que la profesionalización de los cuidados paliativos (el pasaje de los hospices al ambiente medicalizado del hospital) relegó los aspectos psicológicos, sociales y espirituales del cuidado, poniendo en cuestión los principios originales del movimiento. Otras críticas, por su parte, señalan que los cuidados paliativos suponen la expansión de la esfera del control médico a áreas que exceden lo puramente fisiológico ${ }^{26-29}$. Desde esta óptica, el abordaje "integral" de los cuidados paliativos, centrado en la totalidad "bio-psico-social", supone un nuevo horizonte de intervención por parte de la medicina, en que la esfera privada e íntima de la persona, su historia, sus emociones, las relaciones familiares y su relación con la enfermedad y la muerte se configuran como objeto de intervención profesional.

Ahora bien: ¿̇en qué medida los cuidados paliativos "humanizan" el final de la vida o suponen un avance de los procesos de medicalización? ¿En qué medida estos términos pueden presentarse como opuestos? El artículo aborda estos interrogantes y plantea la imposibilidad de observar separadamente estos procesos, mostrando los cruces, tensiones y solapamientos entre prácticas tendientes a "humanizar" la atención, la rutinización de esos cuidados y la medicalización del proceso de morir.

Una de las entradas posibles para analizar las tensiones entre los procesos mencionados en este tipo de servicios es focalizar en las prácticas de cuidado. Las dimensiones del cuidado (como opuestas a las dimensiones técnicas) son aspectos poco valorados de los profesionales de la salud sobre su propia práctica ${ }^{30}$, y usualmente desatendidas o invisibilizadas en los análisis sociológicos y antropológicos de los abordajes médicos. En el artículo se describe la configuración de un cuidado personalizado e integral, en la base de la propuesta de la disciplina de brindar una atención más "humana" en el final de la vida, y analiza la forma en que éstas prácticas están mediadas por el avance de los profesionales sobre áreas de la intimidad de las personas, en las que el cuidado es considerado menos un aspecto "no profesional” de la práctica médica que un área de atención sobre la que se afirman habilidades y saberes técnicos específicos. 


\section{Metodología}

La investigación que da pie al artículo se inscribe en la línea de estudios que aborda la organización social de la muerte en contextos médicos, y que examina las prácticas profesionales e institucionales que constituyen y definen el proceso de morir en estos ámbitos, como hospitales generales $^{31-33}$, unidades de cuidados intensivos ${ }^{5,6,34-36}$ y servicios de cuidados paliativos ${ }^{25,27,28}$.

Los datos analizados en este artículo forman parte de una investigación realizada en un servicio de cuidados paliativos de un hospital público de la ciudad de Buenos Aires, Argentina. El trabajo de campo consistió en observaciones etnográficas en el servicio durante un periodo de trece meses, realizadas en diversas etapas durante los años 2006 y 2009; y en entrevistas semi-estructuradas y charlas informales con profesionales y pacientes.

El trabajo de campo etnográfico supuso la participación en diferentes espacios y situaciones, como reuniones de equipo, pases de sala, y consultas médicas con pacientes y familiares, entre otros. Asimismo, se realizaron entrevistas semiestructuradas con 24 profesionales (médicos, psicólogos y profesionales de enfermería) y 30 pacientes. El material etnográfico fue registrado en un cuaderno de campo y junto con el texto desgrabado de las entrevistas codificado en diferentes dimensiones a partir de un análisis inductivo ${ }^{37}$.

La elección de realizar la investigación en este servicio se debió tanto a cuestiones relativas a la factibilidad (no menores en proyectos de este tipo) y a la relevancia del caso, un servicio pionero de la disciplina y referente en el país y en América Latina. Dado que el principal interés de la investigación fue analizar la gestión del final de la vida en este tipo de servicios, las características reseñadas responden al criterio de relevancia que debe atenderse en la elección del setting en los estudios de caso ${ }^{38}$.

Las entrevistas y observaciones se realizaron con el consentimiento de pacientes y profesionales, informados del carácter y los objetivos de la investigación, que contó con la autorización de los responsables del servicio y con la aprobación de los comités de Ética y de Docencia e Investigación del hospital. Los nombres de los pacientes y los profesionales utilizados en el artículo son seudónimos, y se han omitido o modificado detalles para proteger el anonimato de los participantes.

\section{Resultados}

A continuación se analiza la filosofía y las prácticas de un cuidado integral, base de la propuesta de "humanizar" la atención en el final de la vida. Abordar estas prácticas permite observar la construcción de una mirada clínica sobre el proceso de morir en clave "paliativa", en que el cuidado es considerado menos un aspecto no profesional de la práctica médica que un área de atención sobre la que se afirman habilidades y saberes técnicos, y que expanden las áreas de intervención profesional en el final de la vida.

\section{Cuidado personalizado}

El cuidado, asociado a actitudes de compasión y empatía, se distingue del conocimiento y habilidades técnicas de la biomedicina, en las que queda subsumido ${ }^{39,40}$. En enfermedades crónicas, como en el final de la vida, estos aspectos de la práctica - la contención, la escucha, las relaciones interpersonales entre profesionales y pacientes-adquieren mayor relevancia y, en ocasiones, constituyen un espacio privilegiado de la atención médica.

En una de las etnografías pioneras sobre la atención del morir en el hospital, Sudnow ${ }^{32}$ describe una serie de prácticas consistentes en el tratamiento de los pacientes moribundos como si ya estuviesen muertos. La noción de "muerte social" da cuenta de esas acciones (o inacciones) que privan de individualidad y dignidad a los pacientes en el final de sus vidas. En las antípodas de esas prácticas, el abordaje de los profesionales de cuidados paliativos apunta a construir un cuidado personalizado, que busca respetar la individualidad y las preferencias de los pacientes. La escucha y la contención, las muestras de afecto y consideración con los pacientes, acercarse y tomar la mano de un paciente que llora en la consulta, interactuar con la familia, forman parte de las intervenciones cotidianas por parte de los profesionales, que apuntan a la construcción de un cuidado personalizado. En la sala de internación, por ejemplo, se busca atender a algunos detalles que mejoren la estadía de los pacientes, como indagar en sus preferencias musicales, o en las actividades que les gustaría realizar, de modo de procurar satisfacerlas. Así, los profesionales invitan a una paciente a que cuide las plantas de los canteros de la galería, o se le facilitan materiales para que pueda pintar en su habitación.

Estas prácticas son valoradas muy positivamente por los pacientes, quienes al describir la atención recibida destacan "la calidez en el trato", "el cariño", "la preocupación", "la contención", entre otras expresiones, generalmente contrapuestas a la atención recibida previamente. Como señala un paciente entrevistado: 
P: ¿Y hubo algo de la atención que te sorprendiera?

R: O sea, me sorprendió y no me sorprendió, porque tenía referencias de que era muy bueno el servicio. Pero de todas formas me sorprendió gratamente que se corroborara eso, ¿no?, y que fuera muy bueno el servicio en el sentido de haberme sentido muy bien, muy contenido por los profesionales que me atendieron. Contenido en el sentido de ser comprendido, de tener alguien que del otro lado te está escuchando, está interesado en escuchar, en comprender el padecer que uno trae, y en tener interés en ayudar. Que son quizás cosas básicas, que cualquier médico debiera tener, pero no siempre, por lo menos en mi experiencia yo no lo he visto, ni de los médicos ni de otros profesionales vinculados a la salud, y que acá yo lo noté muy, muy claramente, la existencia de este interés por el paciente, por la persona. (Entrevista a Ariel, paciente)

En oposición a la indiferencia o la "frialdad" de la atención recibida en otros servicios, la calidez y la preocupación por cuestiones que van incluso más allá de lo clínico son rescatados por los pacientes y familiares, y constituyen un aspecto central de la expertise de la disciplina.

\section{El cuidado como expertise técnica}

James ${ }^{41}$ distingue el cuidado personalizado de los hospices - que compara al cuidado familiar, no rentado - del cuidado despersonalizado y profesional del hospital. Estas prácticas de cuidado - personalizado, contenedor, respetuoso del paciente - son reconfiguradas en esta disciplina como una competencia técnica. El manejo de la comunicación y de las emociones, la relación con los pacientes y los familiares, entre otros, son objeto de una preparación y una expertise específica, y constituyen una parte importante de la formación de los profesionales de esta disciplina.

Good y DelVecchio Good ${ }^{39}$ distinguen la competencia y el cuidado como aspectos que cruzan la práctica médica, la primera asociada al lenguaje, conocimiento y habilidades de la medicina, y el segundo a las actitudes de compasión y empatía. En cuidados paliativos ambos aspectos se integran en un saber técnico y en un discurso experto sobre la muerte y el morir, así como del trato con los pacientes y los familiares en esta situación.

La referencia a que no todo abordaje compasivo en el final de la vida puede ser definido estrictamente como "cuidados paliativos", escuchado en congresos de la especialidad y por los profesionales del equipo, subraya esta idea: la configuración de estos cuidados no remite meramente a una atención dedicada o con "buena volun- tad", sino al manejo de competencias técnicas tanto médicas como comunicacionales - en el final de la vida.

Los manuales de la disciplina, además de los capítulos dedicados al manejo del dolor y de los síntomas clínicos de este tipo de pacientes, contemplan los aspectos comunicacionales de la atención. Allí se presentan protocolos para el manejo de la información, para anunciar las malas noticias y contener a los pacientes y a los familiares; $y$ se subraya la importancia del lenguaje gestual, del contacto visual, el tono de voz y el contacto físico (sujetar la mano, tocar al paciente), como formas de "evitar la distancia" y "demostrar interés" en los pacientes.

El contacto físico, la escucha, acompañar a los pacientes en estos momentos difíciles, de mucha angustia y preocupación, supone a su vez un trabajo sobre las emociones o, como señala James ${ }^{41}$, un "trabajo emocional", que también requiere entrenamiento, conocimientos y habilidades técnicas y comunicacionales. La derivación de pacientes por otros servicios del hospital, que en ocasiones convocan a los profesionales de la Unidad para ver un paciente con mucho "sufrimiento emocional", o para gestionar la información sobre un mal pronóstico, o por familiares con un posible "duelo complicado", da cuenta de la dificultad de la medicina para lidiar con estos aspectos emocionales de la práctica médica ${ }^{42}$, y del reconocimiento de la expertise sobre estos aspectos en cuidados paliativos. Las entrevistas en las que los hijos de una paciente consultan a los profesionales acerca de cómo y qué conviene decirle a su madre que está muriendo, o los mismos profesionales aconsejando a los familiares sobre cómo manejar el diálogo sobre temas sensibles, son ocasiones en que los cuidados paliativos se legitiman como un saber autorizado sobre el final de la vida.

\section{El cuidado integral}

El interés por la "persona", señalado tanto por pacientes como por los profesionales de cuidados paliativos, se diferencia de los abordajes más biologicistas en que el paciente es reducido a una patología. El abordaje "integral" u "holista" de la disciplina, expresado en el concepto de "dolor total"26, propone integrar a las dimensiones físicas los aspectos familiares, sociales y espirituales. Como señala una profesional del equipo entrevistada:

Me parece que sí veo, de parte del equipo [de cuidados paliativos], una comunicación diferente. O sea, si yo escucho un pase de un médico en Clínica Médica te va a describir toda la parte médica, te va a describir con detalle qué es lo que está 
pasando en su metabolismo, o qué estudios hizo, qué encontró, qué tipo de célula cancerosa es, y si yo escucho un médico acá te va a contar cómo está compuesta la familia, si tiene apoyo o no, cómo se siente, si está angustiado, si pudo comer. O sea, además de la parte de la metadona, no sé qué medicamento le dan, tiene una visión mucho más amplia y eso, evidentemente es porque tienen otro tipo de comunicación. (Entrevista a María, terapista ocupacional)

Este fragmento describe el enfoque con que trabajan los profesionales de cuidados paliativos, divergente al de otros servicios del hospital. Esta característica, que conlleva elementos positivos en la atención y valorados por los pacientes (la preocupación por la "persona" y la personalización de los cuidados), a la vez amplía el horizonte de intervención profesional. Como señala una médica entrevistada:

En general, cuando vienen los pacientes a cuidados paliativos, la primera evaluación la hace el médico, y después de acuerdo a esa evaluación que es multidimensional, venga por lo que venga, nuestra historia clínica es una historia clínica que evalúa todos los aspectos del paciente en todas las falencias, en todas las áreas, y de acuerdo a eso se hace como un preinforme digamos, de cuáles serían las necesidades del paciente, del área física, del área psicológica. (Entrevista a Ana, médica)

La conformación de este abordaje "integral" es parte del aprendizaje y la formación de los profesionales en la disciplina, lo que supone construir un tipo particular de mirada clínica. Indagar sobre la conformación de la familia, las actitudes con respecto a la enfermedad, los temores, es parte del trabajo cotidiano en las consultas. En palabras de una paciente:

Porque yo cuando vengo a consulta no solamente es consulta médica, es psicológica también, porque les cuento todo lo que pasa en mi vida, y todo tiene una relación aparte. Porque siempre que hay un dolor, o una descompensación, no solamente a veces tiene un motivo físico, quizás es que me falta un mineral, o una vitamina, pero también puede pasar que estoy triste, o que tuve un problema en casa con mis hijas, o que tuve un berrinche o que me enojé o lo que fuera, entonces a veces es por una cosa que no es física, y todas esas cosas se contemplan cuando venís a la consulta. (Entrevista a Fernanda, paciente)

En la práctica, esta mirada implica desarrollar competencias para recabar y registrar información relevante sobre las diferentes cuestiones, a partir de la que se construye un conocimiento íntimo sobre el paciente ${ }^{27}$. En este trabajo colaboran todos los profesionales que participan en el seguimiento (médicos, psicólogos, enfermeros, voluntarios). En palabras de una enfermera del equipo:

En otros lados a los pacientes se les baja la persiana mucho antes. Acá no, acá la bajan ellos cuando pasan el umbral. En otros servicios no hay espacio para abrirse, para una mayor empatía con los pacientes: es como que tienen un escudo, que no se quieren dar cuenta, no quieren enfrentar lo que tienen. Como que no quieren ver. Acá no, acá es distinto. Acá podés llegar más profundo, hablar con la gente, comunicarte y dar mucho amor. Yo sentía que tenía mucho más para dar, y acá puedo hacerlo. Yo siento que acá puedo dar todo. Acá se puede hablar, los pacientes acá largan todo, hasta lo que no quieren. Si no es un enfermero el que entra a la habitación es un médico, o la psicóloga, siempre hay alguien que está preguntando. (Notas de campo, conversación con Liliana -enfermera)

El pasaje citado ilustra el modo en que las prácticas de cuidado están atravesadas por la puesta en acto de esta mirada "integral", a partir de la cual el equipo busca identificar "falencias", problemas o vulnerabilidades en la experiencia de los pacientes y familiares, quienes son convocados a colaborar en este trabajo:

$Y$ hay algunos pacientes que niegan la enfermedad, entonces bueno, la esconden, no quieren saber nada de la enfermedad, la esconden y la niegan. Son los pacientes que más ayuda psicológica necesitan. Porque sí... uno tiene que saber lo que le está pasando para poder ayudarlo. Si el paciente no habla, niega todo, y no se expresa, o sea, sentimos que cómo podemos ayudarlos, entonces hay que ver qué le está pasando para que pueda sacar todo eso, quizás temor, dolor, ansiedad, angustia, diversos problemas que está pasando, y bueno, tiene que sacarlos afuera para poder ayudarlo uno, ¿no? Muchas áreas en las que uno lo puede ayudar. (Entrevista a Rosa, enfermera)

Como se desprende de este pasaje, el abordaje holista de los cuidados paliativos no sólo implica una preocupación por la "persona", en el sentido de comprender el sufrimiento desde una perspectiva que exceda el biologicismo que caracteriza y define a la medicina occidental o biomedicina ${ }^{42,43}$. Abordaje que comporta asimismo una "transformación profesional de la experiencia" ${ }^{\text {"44 }}$, en tanto esa mirada "integral" está orientada a diagnosticar otros "síntomas" que también requieren de las intervenciones del equipo. Las respuestas afectivas de los pacientes ante esta situación, las relaciones familiares, entre otras cuestiones, son traducidas como recursos, capacidades, obstáculos y vulnerabilidades del paciente y su entorno, cuya evaluación orientará el tratamiento por parte de 
los profesionales. Un paciente "negador", una familia "poco continente", requerirán de intervenciones puntuales por parte del equipo. El pasaje de la entrevista a una médica de la Unidad muestra los alcances difusos que están implícitos en este abordaje integral, guiado por la preocupación genuina por el bienestar del paciente:

Otro punto [de la atención en cuidados paliativos] es tener en cuenta a la persona entera, ¿no? Y a la familia sobre todo. Mucha más relación y conocimiento de lo que es la familia, el medio donde el paciente vive. A veces para otras cosas no te es tan necesario porque con asegurarte de que reciba cierta medicación o de que lo tengan contenido en cierto lugar al paciente te alcanza. En cambio acá es como que necesitás conocer todo porque la vida del tipo, como que su calidad de vida va a depender de ese entorno. Entonces vos tenés que ver quién lo va a cuidar, cómo lo va a cuidar, qué cosas hay que modificar y todo eso. (Entrevista a Carla, médica)

Ahora bien, ¿qué supone -como refiere la médica entrevistada- "conocer todo" de la vida de un paciente? ¿En qué intervenciones se traduce esta preocupación sincera y bienintencionada por mejorar la calidad de vida del paciente? Una médica señala sus dudas respecto a cuáles deberían ser los límites del abordaje "integral", al tiempo que describe las intervenciones eminentemente normativas realizadas por el equipo sobre el paciente y la familia:

$\mathrm{P}:$ ¿Cómo pensás que es concretamente la atención en cuidados paliativos acá y cómo debería ser?

R: Bueno acá lo veo como que es bastante obsesiva, este y por un lado me parece bien, se intenta estar en casi todos los detalles que se puedan, ¿no? Hay veces, a mi modo de ver las cosas creo que van mucho más allá de lo que para mí serían mis límites. Hay veces que creo que se entra demasiado en las cosas que para mí son demasiado intimas de la familia, o de las personas y no sé hasta qué punto, yo todavía sigo teniendo la duda hasta qué punto uno se puede seguir metiendo tanto, ¿no? Hay familias que funcionan de una determinada forma $y$ este, no sé si decir normal o no, pero frecuentes a la forma de manejarse, de funcionar y uno no tiene por qué siempre tener que meterse a transformarla en lo que uno quiere. Hay veces que esas familias por ahí funcionan desadaptadamente y uno las quiere corregir y uno no tiene por qué siempre estar metiéndose y eso es lo que veo que pasa, que muchas veces se mete, se intenta corregir cosas que no funcionan bien, pero no sé hasta qué punto se debe hacer eso y bueno, o sea ese es el tema de los limites que estamos todo el tiempo hablando de eso, porque uno se mete tanto, es tan obsesivo con tantos detalles que a veces se pasa de largo. Pero bueno, así es como tampoco se van de largo muchos detalles que me parece que a veces son imprescindibles. (Entrevista a Corina, médica)

Como lo ilustra el fragmento, el abordaje integral y las nociones de la disciplina sobre las formas adecuadas de la culminación de una vida, representadas por el ideal de "buen morir"27,28,45, movilizan intervenciones potencialmente normativas por parte de los profesionales, tanto acerca del funcionamiento de las familias como de modos "saludables" de hacer frente y sobrellevar esta experiencia.

Escuchar al paciente, indagar en sus relaciones y sus temores, no supone necesaria o únicamente asumir el compromiso de tratar la totalidad de la "persona", los intereses y sentimientos del paciente, su individualidad y su contexto, como señala Krakauer ${ }^{22}$. En definitiva, de atender (no en un sentido clínico) al sufrimiento del otro sin objetivarlo. En la práctica, este abordaje es asimismo un medio para detectar "síntomas", problemas y obstáculos en la experiencia de los pacientes - "las falencias en todas las áreas" referidas por una médica- como una forma de construir y legitimar espacios de intervención profesional, que medicaliza la experiencia del morir en un sentido más amplio. Ya no sólo son atendibles (ahora sí en sentido clínico) el dolor y los síntomas en el final de la vida, sino la elaboración que el paciente hace de su propia muerte, pasible de ser catalogada como "patológica", o las relaciones que se entablen entre los familiares a partir de esta situación ${ }^{46}$. La mirada integral supone, pues, evaluar los recursos, las dificultades, las potencialidades y vulnerabilidades de pacientes y familias.

\section{Conclusiones}

En este artículo se analizaron la filosofía y las prácticas de un cuidado integral, base de la propuesta de humanización de la atención del final de la vida en cuidados paliativos. Los cuidados paliativos, sus discursos y prácticas, al tiempo que un intento de "humanización" de la atención, suponen el avance de procesos de medicalización en el final de la vida; procesos que no deben entenderse necesariamente como opuestos, sino como aspectos complementarios del fenómeno en cuestión.

En las páginas previas se dio cuenta de prácticas identificadas con un cuidado "más humano" en el final de la vida, dimensión usualmente invisibilizada en los análisis de las prácticas médicas. Se analizó la configuración de un cuidado personalizado, basado en la escucha, la conten- 
ción y el manejo de la comunicación, que apunta a valorar la individualidad y la autonomía de los pacientes, y a desarrollar una aproximación compasiva al sufrimiento. La propuesta de una atención "más humana" se apoya en un abordaje "integral", que incorpora aspectos que exceden lo estrictamente médico como forma de superar el reduccionismo biologicista de considerar a la persona como una patología orgánica. Las referencias de los pacientes a sentirse tratados como "personas", "contenidos", se inscribe en buena medida en las coordenadas de este enfoque.

Estas prácticas de cuidado no suponen un proceso de "desmedicalización”, sino la afirmación de habilidades técnicas específicas y de nuevos especialistas. Si los primeros trabajos empíricos sobre la gestión de la muerte en el hospital señalaban la falta de preparación para lidiar con los aspectos emocionales y la comunicación ${ }^{31-32}$, el abordaje de la organización médica del final de la vida en cuidados paliativos permite observar cómo, amén de nuevas competencias técnicas en el área clínica (como el manejo de múltiples y variados síntomas, entre los que se destaca el dolor), la disciplina reclama su expertise en estos aspectos a priori "no técnicos" de la práctica médica.

El abordaje "integral" expande las esferas de actuación profesional, que pueden dar lugar a intervenciones normativas sobre la experiencia de morir. Los sentimientos y temores, las relaciones y dinámicas familiares, entre otros aspec- tos, son pasibles de ser evaluados y reconvertidos en objeto de intervenciones específicas: las experiencias de los pacientes y de los familiares son traducidas como recursos, obstáculos y vulnerabilidades en el trabajo de producción del "buen morir".

La medicalización del final de la vida operada por los cuidados paliativos no supone, en sí misma, una imputación, como se plantea desde algunas perspectivas críticas. Estos procesos pueden ser positivos o negativos, o ambas cosas, dependiendo de la situación y el contexto. En ese sentido, las lógicas de humanización y del avance de la medicalización en el final de la vida no debieran entenderse como fenómenos opuestos: el artículo buscó evidenciar cómo ambos procesos se armonizan y tensionan, se complementan y potencian.

Desde una perspectiva sociológica, tal como apunta Conrad ${ }^{47}$, debería interrogarse a los procesos de medicalización no en su validez, es decir en qué medida tal fenómeno amerita ser definido o no en términos médicos o profesionales, sino en las condiciones que lo hacen posible: las situaciones y contextos que legitiman o avalan, en este caso, la afirmación de un saber experto sobre el final de la vida. En este sentido, más que una respuesta a los problemas que plantea el morir en ámbitos médicos, la emergencia de esta disciplina revela el carácter problemático y el desafío que supone la gestión del final de la vida en estos espacios.

\section{Agradecimientos}

La investigación se financió con una beca doctoral del Consejo Nacional de Investigaciones Científicas y Técnicas (2006-2011). Agradezco a los profesionales del servicio y en especial a los pacientes y sus familiares: sin su predisposición y buena voluntad para permitirme compartir sus experiencias la investigación no hubiera sido posible.

\section{Referencias}

1. Illich I. Némesis Médica: La expropiación de la salud. México: Joaquín Mortiz; 1978.

2. Ariès Ph. El hombre ante la muerte. Madrid: Taurus; 1992.

3. Ariès Ph. Morir en Occidente: desde la Edad Media hasta nuestros días. Buenos Aires: Adriana Hidalgo; 2000.

4. Seale C. Changing patterns of death and dying. Soc Sci Med 2000; 51:917-930.

5. Menezes RA. Difíceis decisões: uma abordagem antropológica da prática médica em CTI. Physis 2000; 10(2):27-49.

6. Seymour J. Critical moments. Death and dying in Intensive Care. Buckingham: Open University Press; 2001.

7. Gherardi C. Vida y muerte en terapia intensiva. Buenos Aires: Biblos; 2007.

8. Lock M. Twice dead: organ transplants and the reinvention of death. Berkeley: University of California Press; 2002. 
9. Gorer G. Death, grief and mourning in contemporary Britain. London: Cresset; 1965.

10. Elias N. La soledad de los moribundos. México: FCE; 1989.

11. Mellor PA. Death in high modernity: the contemporary presence and absence of death. In: Clark D, editor. The Sociology of Death. Oxford: Blackwell; 1993. p. 11-30.

12. Mellor PA, Schilling CH. Modernity, self-identity and the sequestration of death. Sociology 1993; 27(3):411-431.

13. Walter T. The revival of death. London: Routledge; 1994.

14. Clark D, Seymour J. Reflections on palliative care: sociological and policy perspectives. Buckingham: Open University Press; 1999.

15. Emmanuel E. Euthanasia: historical, ethical and empiric perspectives. Arch Intern Med 1994; 154(12): 1890-1901.

16. McInerney F. "Request death": a new social movement. Soc Sci Med 2000; 50:137-154.

17. Rothman D. Strangers at the bedside: a history of how law and bioethics transformed medical decision making. New York: Basic Books; 1991.

18. Williams S, Calnan M. The 'limits' of medicalization?: modern medicine and the lay populace in 'late' modernity. Soc Sci Med 1996; 42(12):1609-1620.

19. Lupton D. Consumerism, reflexivity and the medical encounter. Soc Sci Med 1997; 45(3):373-381.

20. World Health Organization (WHO). National cancer control programmes: policies and managerial guidelines. Ginebra: WHO; 2002.

21. Wainer R. "Yo no te puedo decir cuánto sino cómo": Construcción y manejo de la esperanza en el final de la vida, desde la perspectiva profesional de un equipo de cuidados paliativos. Anuario de Estudios en Antropología Social; 2004; p. 187-197.

22. Krakauer E. 'To be freed form the infirmity of (the) age': subjetivity, life-sustaining treatment, and palliative medicine. In: Biehl J, Good B, Kleinman A, editores. Subjetivity. Ethnographic Investigations. California: University of California Press; 2007. p. 381-396.

23. James N, Field D. "The routinisation of hospice: charisma and bureaucratisation". Soc Sci Med 1992; 34(12):1363-1375.

24. Bradshaw A. The spiritual dimension of hospice: the secularization of an ideal. Soc Sci Med 1996; 43(3):409-419.

25. McNamara B. Good enough death: autonomy and choice in Australian palliative care. Soc Sci Med 2004; 58(5):929-938.

26. Clark D. 'Total pain', disciplinary power and the body in the work of Cicely Saunders, 1958-1967. Soc Sci Med 1999; 49(6):727-736.

27. Castra M. Bien mourir. Sociologie des soins palliatifs. Paris: PUF; 2003.

28. Menezes RA. Em busca da boa morte: antropología dos cuidados paliativos. Rio de Janeiro: Garamond, Fiocruz; 2004.

29. Zimmermann C. Denial of impending death: a discourse analysis of the palliative care literature. Soc Sci Med 2004; 59(8):1969-1780.

30. Mattingly C. Healing dramas and clinical plots. The narrative structure of experience. Cambridge: Cambridge University Press; 1998.
31. Glaser B, Strauss A. Awareness of dying. Chicago: Aldine; 1965.

32. Sudnow D. La organización social de la muerte. Buenos Aires: Tiempo Contemporáneo; 1971.

33. Epele M. Una aproximación antropológica a la enfermedad terminal. In: Álvarez M, Barreda V, compiladores. Cultura, salud y enfermedad. Temas en antropología médica. Buenos Aires: INAyPL; 1995. p. 157-161.

34. Zussman R. Intensive care: medical ethics and the medical profession. Chicago: University of Chicago Press; 1992.

35. Anspach R. Deciding who lives: fateful choices in the intensive-care nursery. Berkeley: University of California Press; 1993.

36. Harvey J. The technological regulation of death: with reference to the technological regulation of birth. Sociology 1997; 31(4):719-735.

37. Strauss A, Corbin J. Bases de la investigación cualitativa. Técnicas y procedimientos para desarrollar la teoría fundamentada. Medellín: Editorial Universidad de Antioquia; 2002.

38. Stake R. Case Studies. In: Denzin N, Lincoln Y, editores. Handbook of Qualitative Research. California: Sage; 1994. p. 236-247.

39. Good BJ, DelVecchio Good MJ. Learning medicine. The construction of medical knowledge at Harvard Medical School. In: Lindebaum S, Coch M, editores. Knowledge, power and practice. The Anthropology of Medicine and everyday life. California: University of California Press; 1989. p. 81-107.

40. Ayres, J.R. Sujeito, intersubjetividade e práticas de saúde. Cien Saude Colet 2001; 6(1):63-72.

41. James N. Care $=$ organisation + phisycal labour + emotional labour. Sociology of Health \& Illness 1992; 14(4):488-509.

42. Menendez E. Morir de alcohol. Saber e ideología médica. México: Alianza; 1990.

43. Bonet O. Saber e sentir: uma etnografía da aprendizagem da biomedicina. Rio de Janeiro: Fiocruz; 2004.

44. Kleinman A. Writing at the margin. Berkeley: University of California Press; 1995.

45. Alonso JP. Contornos negociados del "bien morir": la toma de decisiones médicas en el final de la vida. Interface (Botucatu) 2012; 16(40):191-203.

46. Alonso JP. Manejo de la información sobre malos pronósticos y psicologización del final de la vida en cuidados paliativos. Acta Psiquiátrica y Psicológica de América Latina 2011; 57(4):267-274.

47. Conrad P. The medicalization of society. On the transformation of human conditions into treatable disorders. Baltimore: Johns Hopkins University Press; 2007.

Artigo apresentado em 30/04/2013

Aprovado em 22/05/2013

Versão final apresentada em 30/05/2013 